\section{Radium and the Evolution of the Earth's Crust.}

IT is now well known that if the proportion of radium in the interior of the earth is the same as that in the surface rocks, the earth ought to be growing hotter, and the temperature gradient of the crust ought to be much higher than we find it. A simple calculation shows that a distribution of radium averaging $2 \times 10^{-14}$ grams in each gram of rock throughout the earth would suffice to preserve thermal equilibrium. The amounts of radium actually found in the surface rocks are approximately as follows :-

\begin{tabular}{|c|c|c|c|c|c|}
\hline Acid & & \multicolumn{4}{|c|}{$3 \times 10^{-12}$ grams per gram } \\
\hline Intermediate & $\ldots$ & 2 & ", & ", & $"$ \\
\hline Basic $\quad \ldots$ & $\ldots$ & I & ," & , & , \\
\hline Ultrabasic & $\ldots$ & 0.5 & ", & $"$ & ,, \\
\hline
\end{tabular}

In addition to the elements of the uranium family, those of the thorium family must also be considered, for they afford an equally important supply of heat.

Prof. Strutt was the tirst to indicate the way in which the obvious dilemma might be escaped. In order that the earth should be nearly in thermal equilibrium (i.e. not growing hotter, but cooling at the very slow rate allowed by the radio-active elements as they decrease in quantity in accordance with their progressive disintegration), it is necessary to assume that the earth's store of radium is concentrated near the surface. As the following arguments indicate, this conception is less arbitrary than would appear at first sight. The radio-active elements are found most abundantly in the acid rocks, their more basic associates being less embarrassingly rich. The more acid rocks are characteristic of only the outermost zones of the crust, and there are many reasons for believing that with depth the more basic rocks largely predominate. Seismic and other terrestrial phenomena have now provided us with data from which the condition of the earth's interior may be deduced with some confidence. First, there is the crustal zone, rapidly becoming less silicic with depth, having a mean density of $2 \cdot 8$, and an approximate thickness of thirty miles. Within a fairly sharp surface of discontinuity comes what may be called the stony zone. The density is 3.4 , and judging from the close analogy presented by meteorites, the material would be of ultra-basic composition. This zone dies out at a depth variously estimated at from 600 to 900 miles. The internal core of the earth is probably largely composed of iron, its density being about 8 .

In a number of meteorites, the radium content has been determined by Prof. Strutt and the present writer, and if it may be assumed that they afford a clue to the problem, the heavy metallic core should be completely destitute of radium, and the stony zone should contain only a small proportion, very much less than that of the ultra-basic rocks of the crust.

On the planetesimal hypothesis, the two internal zones find a ready explanation. It is supposed that the earth began as a nebulous knot, and that it has grown up to its present mass by the capture of associated planetesimals. It is very unlikely that as a whole it was ever in a molten condition. Internal heat probably arose largely from the condensation of the mass during the period of its growth. The temperature would slowly rise until the fusion point of certain of the constituents was reached, and the liquid tongues and pockets thus formed would tend to move away from the centre, the lighter and less viscous stony material being squeezed outwards relatively to a network of the heavier and more rigid metallic materials. Once vulcanism had been initiated in this way, the process would continue until a highly metallic nucleus had collected. Surrounding it there would gradually form a thick zone of silicate rocks, NO. 2277 , VOL. 9I] the differentiation from the original heterogeneous mixture of stony and metallic constituents being due to the selective fusion of the former. There seems to be little doubt that the radio-active elements would be concentrated in the stony zone. With the establishment of ocean and atmosphere, a new factor in surface differentiation arose, and sedimentary rocks were deposited for the first time. In some way which, as yet, we understand but vaguely, both igneous and denudational differentiation then combined in developing the earth's crust. We now find in the latter all those rocks which hold a maximum content both of silica and of the radio-active elements. The relative concentration of these constituents having taken pluce at the expense of the zone below, the conjectural paucity of the latter in radium finds a suggestive explanation.

Before the advent of radium, geologists had not recognised the difficulties presented by the peculiar chemical constitution of the earth's crust. Radium did not create this difficulty, but has merely directed attention towards it. Any explanation of the high percentage of silica in the surface rocks will explain equally well their richness in radium.

It can scarcely be said now that radium has given us "a blank cheque on the bank of time." Not only did the discovery of radium destroy the validity of the older thermal arguments, but also it led directly to the elaboration of a new and more refined method. Every radio-active mineral may be regarded as a selfcontained hourglass, the radio-active end-products, helium and lead, slowly accumulating at the expense of their ultimate parent, uranium. In the few cases which up to the present have been investigated, periods of enormous duration have been revealed, and the geologist who ten years ago was embarrassed by the shortness of the time allowed to him for the evolution of the earth's crust is now still more embarrassed by the superabundance with which he is confronted. The time scale up to date, as determined by the lead ratio, is as follows :-

$\begin{array}{llllll}\text { Carboniferous } & \ldots & \ldots & 340 \\ \text { Devonian } & \ldots & \ldots & \ldots & 370 & , \\ \text { Ordovician } & \ldots & \ldots & \ldots & 430 & " \\ \text { Algonkian } & \ldots & \ldots & \ldots & \begin{array}{r}1000 \\ 1300\end{array} \\ \text { Archæan } & \ldots & \ldots & \ldots & , \\ 1600 & ,\end{array}$

We must not moan over the apparent difficulties with which the geologist has been faced since the advent of radium. Rather should they be welcomed in that they open the way for further advances. If at present some of our ideas are mutually incompatible, the discrepancies do not demand a wholesale rejection of the facts, but simply a re-interpretation of the fundamental hypotheses on which so many of our doctrines seem to hang.

Imperial College, South Kensington, S.W.

\section{An Amphipod Invasion.}

MANY specimens of a small amphipod crustacean, Euthemisto compressa, Goës, have been forwarded to me by Mr. T. H. Nelson, of Redcar. On May 23 and 24 these were washed ashore in incredible numbers on the coast of Yorkshire, where they lay from Saltburn to Teesmouth-a distance of ten miles-in drifts several inches deep. The pools were alive with the crustaceans, and to the east of Redcar a fisherman was seen raking them into heaps, and wheeling away barrow-loads to put on his garden as manure. In the sea hundreds at a time could be scooped up in one's hands.

Euthemisto compressa is an uncommon British 